\title{
Increased Leptin Permeation across the Blood-Brain Barrier after Chronic Alcohol Ingestion
}

\author{
Weihong Pan*,', Misty Barron', Hung Hsuchou', Hong Tu' and Abba J Kastin' \\ 'Blood-Brain Barrier Lab, Pennington Biomedical Research Center, Baton Rouge, LA, USA
}

Leptin, a polypeptide mainly produced in the periphery, crosses the blood-brain barrier (BBB) by receptor-mediated transport to exert multiple central nervous system actions including decreased food intake. The reciprocal interactions between leptin transport and alcohol drinking are not clear. In this study, we tested whether alcohol increases leptin entry into brain and, if this occurs, whether it is a consequence of a generalized increase in the permeability of the BBB. BBB permeability to albumin, the increased permeation of which indicates BBB disruption, as well as to leptin was measured after alcohol ingestion. CDI and B6 mice ingested a 5\% liquid alcohol diet or its isocaloric control for 2 weeks. Alcohol ingestion resulted in increased blood-alcohol levels, decreased blood-leptin concentrations, and increased permeation of radioactively labeled leptin across the BBB as shown by in situ perfusion. Although the increased influx of the vascular marker albumin into brain showed partial disruption of the BBB, the influx of ${ }^{125}$-leptin still could be suppressed by excess unlabeled leptin, indicating persistence of its saturable transport system. When given a choice of either alcohol or control diet, even the alcohol-preferring B6 mice showed a significantly greater preference for the control liquid diet, and there was no evidence of BBB disruption or alterated leptin transport. Furthermore, acute alcohol intoxication induced by intraperitoneal injection of $20 \%$ alcohol did not result in BBB disruption or increased leptin permeation $4 \mathrm{~h}$ later. Thus, partial disruption of the BBB and increased permeation of leptin in both $\mathrm{CDI}$ and B6 mice were only induced by chronic alcohol ingestion. The results showing increased leptin permeation across the BBB lead to the speculation that leptin may serve as a homeostatic feeding signal in these mice.

Neuropsychopharmacology (2008) 33, 859-866; doi: I0. I038/sj.npp. I 301452; published online 9 May 2007

Keywords: alcohol; blood-brain barrier; leptin; transport system; preference; feeding

\section{INTRODUCTION}

Animal models of diet-induced alcoholism reveal a striking difference in alcohol (ethanol, EtOH) preference among different strains of mice (Vavrousek-Jakuba et al, 1991; Blednov et al, 2004). The C57BL/6J (B6) strain of alcoholpreferring mice showed the most consistently high intake of alcohol of 15 inbred mouse strains tested (Belknap et al, 1993). We examined whether the cardinal ingestive peptide leptin participates in the feeding behavior of alcohol preference, especially in the B6 strain.

Leptin is mainly produced by adipose tissue and can cross the blood-brain barrier (BBB) to act on the hypothalamus and other central nervous system (CNS) targets to reduce food intake (Kastin and Pan, 2006). There are complex interactions among alcohol ingestion, blood-leptin concentration, and the CNS response to leptin. Many other factors in addition to leptin can modulate alcohol ingestion, such as

*Correspondence: Dr W Pan, Blood-Brain Barrier Lab, Pennington Biomedical Research Center, 6400 Perkins Road, Baton Rouge, LA 70808, USA, Tel: + I 225763 2707, Fax: + I 225763 026I,

E-mail: weihong.pan@pbrc.edu

Received 27 September 2006; revised 26 January 2007; accepted 13 April 2007 methionine-enkephalin (Banks et al, 2006b; Urayama et al, 2006). Nevertheless, there is evidence that leptin participates in the metabolic disturbance associated with chronic alcoholism, shown by the effect of leptin in improving insulin resistance induced by chronic administration of high doses of alcohol in mice (Balasubramaniyan and Nalini, 2006).

Various studies have found an increase, decrease, or no change in blood concentrations of leptin after alcohol ingestion, depending upon test conditions. Circulating leptin levels do not correspond with the amount reaching the brain by BBB transport (Banks et al, 2000). The transport system is saturable and has limited capacity (Banks et al, 1996; Kastin et al, 2001). Thus, the apparent influx rate of leptin may be altered by its concentration in blood (Kastin et al, 1999; Banks et al, 2002). In this study, the direct effects of alcohol on the transport system for leptin at the BBB were determined after in situ perfusion in blood-free buffer.

Mild alcohol intoxication was induced in mice by a liquid diet containing $5 \%$ alcohol for 2 weeks after gradual adaptation. In contrast to this forced feeding regimen in which alcohol was the only source of energy intake, we also conducted a two-bottle choice feeding study. In addition, 
the effect of acute alcohol administration was tested to determine whether alcohol dependence is required for altered leptin influx. In this way, the experimental variables of duration of alcohol exposure, and voluntary compared with forced drinking, were examined.

\section{MATERIALS AND METHODS}

Young adult male mice (5-7-week-old) of either CD1 or B6 strains were used for the study. The animal protocol was approved by the Institutional Animal Care and Use Committee. Recombinant mouse leptin was obtained from Calbiochem (Temecula, CA). Bovine serum albumin, chloramine-T, sodium metabisulfite, and Sephadex G-10 were obtained from Sigma (St Louis, MO). ${ }^{125} \mathrm{I}$ was purchased from Amersham-GE Sciences (Piscataway, NJ) and ${ }^{131} \mathrm{I}$ was purchased from PerkinElmer (Boston, MA). Radioactive labeling (radiolabeling) and purification of ${ }^{125} \mathrm{I}$ leptin and ${ }^{131} \mathrm{I}$-albumin were performed by the chloramine$\mathrm{T}$ method, and Sephadex G-10 columns as described previously. The 190 proof alcohol used for the feeding studies was obtained from Pharmco Products (Brookfield, CT). Powdered food to prepare the liquid diets was obtained from Bio-Serv (Frenchtown, NJ). The liquid diet offers $1000 \mathrm{kcal} / \mathrm{l}$ of calorie, with a distribution of $208 \mathrm{kcal} / \mathrm{l}$ from protein, $119 \mathrm{kcal} / 1$ from fat, and $673 \mathrm{kcal} / 1$ from carbohydrate.

In the forced alcohol-feeding studies, a group of mice 6-8-week-old were adapted to a nonalcoholic liquid diet for 3 days and then gradually adapted to an alcoholic liquid diet starting with $1.67 \%$ alcohol for 3 days, followed by $3.94 \%$ alcohol for another 3 days. The mice were then given a 5\% alcohol-containing diet for 14 days. The control group was fed an isocaloric liquid diet. Study of both B6 and CD1 mice not only provides new data on strain differences, but it also facilitates comparison with previous reports as most leptin transport assays have been conducted on CD1 mice.

In the two-bottle pair-feeding studies, a 5\% alcohol and a control isocaloric liquid diet were freely available to mice for 14 days after the 3 days initial adaptation period to liquid diet. To control for place preference, the alcohol and water bottles were alternated every 2 days. Alcohol and water consumption and body weight were monitored regularly. Control cages without mice were included in the study to exclude errors caused by unwanted motion or leakage. Only B6 mice were used for this study, as preliminary data showed that CD1 mice have an even lower preference for alcohol.

The acute alcohol model was generated by intraperitoneal (i.p.) injection of $20 \%$ alcohol $(2 \mathrm{~g} / \mathrm{kg}$ of body weight $) 4 \mathrm{~h}$ before the $\mathrm{BBB}$ transport assay. The control group received vehicle $(0.9 \% \mathrm{NaCl})$ injected at the same time ( $n=6$ /group). This study design involved both B6 and CD1 mice to test for potential differences in their $\mathrm{BBB}$ transport of leptin to the influence of alcohol.

No significant adverse effects of alcohol ingestion, such as hypothermia, failure to thrive, unusual body tremor, fur muffling, or seizures, were observed in any of the studies. However, discoordination in beam walking and altered locomotor activity were noticed in mice 2 weeks after forced alcohol feeding or within the first hour of i.p. injection of alcohol.

At the end of the feeding studies or after i.p. injection of alcohol, the mice were anesthetized by i.p. injection of a mixture of ketamine/xylazine/acepromazine and used either for measurement of blood concentrations of alcohol and leptin or for transport measurements. To avoid withdrawal, alcohol feed was present until the mice were anesthetized, and they were studied within $10 \mathrm{~min}$ of anesthesia.

The concentration of leptin in serum was measured by a quantitative sandwich enzyme immunoassay (MOB00, R \& D Systems, Minneapolis, MN). The assay sensitivity was $22 \mathrm{pg} / \mathrm{ml}$. The concentration of alcohol was measured by an alcohol dehydrogenase/reduced nicotinamide dinucleotide assay (Sigma) in serum stored in tightly sealed vials at $-80^{\circ} \mathrm{C}$ until use. A standard solution of alcohol was used to generate the standard curve. Assays of leptin and alcohol concentrations involved samples obtained in different mice from those used for BBB transport assays, but were age- and sex-matched and fed at the same time.

Permeability of the BBB to the vascular marker albumin and leptin was determined by in situ brain perfusion of radiotracers as described previously (Pan et al, 1998). Both a single-time point study design (5 min of perfusion with radiotracers) and a multiple-time regression study design were used. In brief, $1 \%$ bovine serum albumin was added to the oxygenated perfusion buffer and the $\mathrm{pH}$ adjusted to 7.4. The perfusion was driven by a syringe pump at a speed of $2 \mathrm{ml} / \mathrm{min}$. Each anesthetized mouse was transcardially perfused with buffer for $2 \mathrm{~min}$ to remove blood from the vasculature. This was followed by perfusion containing both ${ }^{125} \mathrm{I}$-leptin and ${ }^{131} \mathrm{I}$-albumin $(0.5 \mu \mathrm{Ci} / \mathrm{ml}$ of each $)$ for the designated duration, after which the mice were again perfused with buffer for 1 min to remove any radiotracers remaining in the cerebral blood vessels. Two types of statistical analyses were performed: comparison of uptake at a single time point (mean $\pm \mathrm{SE}$ ) by analysis of variance, and comparison of the difference in the influx rate $\left(K_{\mathrm{i}}\right)$ by multiple-time regression studies. The analyses were performed with SPSS and GraphPad Prism Statistical Analysis software.

To test the saturability of leptin influx at the end of the alcohol feeding, excess unlabeled leptin was included either in the in situ brain perfusion buffer or in the bolus intravenous (i.v.) injection, as specified in the Results. ${ }^{125} \mathrm{I}$ leptin was injected i.v. into anesthetized mice in a bolus of $100 \mu \mathrm{l}$ of lactated Ringer's solution containing $1 \%$ bovine serum albumin through the jugular vein at time 0 . The mice were decapitated at various times, $1-20 \mathrm{~min}$ after i.v. injection. The linear regression correlation between the brain/blood ratio and the exposure time was used to calculate the influx rate $K_{\mathrm{i}}$ as described previously. In this assay, groups of mice (at least 7/group) were studied, each representing a different time point. The $K_{\mathrm{i}}$ values were compared by analysis of variance to determine whether there were differences within groups. This was followed by Newman-Keul's post hoc test to determine which values within a group differed. The standard deviation of the mean for the slope was taken as the standard error of the mean and, because two means (the slope and the intercept) were calculated from the data, $n-1$ was used as the $n$-value in the analysis of variance and range tests. Significant differences 
between groups were determined with the aid of the GraphPad Prism statistical program (Kastin and Pan, 2003; Pan et al, 2004).

\section{RESULTS}

Feeding Behavior and Blood Concentrations of Alcohol and Leptin in Mice on a Forced Alcohol Diet

After 9 days of adaptation to the liquid diet and gradual increase of alcohol concentration, groups of mice $(n=12-$ 14 /group) were fed either a $5 \%$ alcohol diet or an isocaloric nonalcohol diet for 2 weeks. Despite the 9-day adaptation period, both B6 and CD1 mice showed reduced alcohol intake when the alcohol concentration was increased to 5\%. Although the formula for the alcohol and control liquid diet groups contained similar composition and the same energy supply $(\mathrm{kcal} / \mathrm{ml})$, the alcohol groups drank less and thus had lower daily caloric intake. This reduced alcohol intake persisted for the entire 14 days in B6 mice, but only lasted a week in the CD1 mice (Figure 1a). Correlating with the reduced intake of liquid food, there was a significant reduction in body weight that was more pronounced in B6 mice than in CD1 mice (which weighed more than the agematched B6 mice even in the control groups) (Figure 1b). The stepwise increase of blood-alcohol concentration was apparent in CD1 mice, as shown in the line graph (Figure 1c). The B6 mice, studied at a different time, appeared to show higher blood-alcohol concentrations after 14 days, which dropped precipitously 2 days after withdrawal (Figure 1c, inset).

There was no significance difference in water consumption in groups of mice fed with control diet or alcohol (Figure 1d). Nonetheless, concurrent with the significant increase of alcohol levels was a significant decrease in blood-leptin concentrations when measured at day 14 (Figure 1e). These lower blood-leptin concentrations were consistent with the lower body weights of the alcohol groups compared with the control groups fed an isocaloric diet. Thus, alcohol feeding significantly increased bloodalcohol and decreased blood-leptin concentrations in both strains of mice $(P<0.001)$.

The Effect of Forced Alcohol Diet on the Blood-to-Brain Influx of Albumin (Vascular Marker) and Leptin (Polypeptide with Specific Transport System) in Nonalcohol-Preferring CD1 Mice

As the groups of mice fed alcohol had significant differences in body weight and blood-leptin concentrations from the control groups, the potential use of i.v. injection to evaluate BBB permeability could be confounded by peripheral binding proteins and endogenous leptin levels. Therefore, in situ brain perfusion was performed to test the regression correlation between the brain/perfusate ratio and perfusion time (1-10 min). The influx rate of ${ }^{125}$ I-leptin in CD1 mice was $0.47 \pm 0.18 \mu \mathrm{l} / \mathrm{gmin}$ in the control group $(n=8)$ and $1.24 \pm 0.26 \mu \mathrm{l} / \mathrm{gmin}$ in the alcohol group $(n=7)$. The difference between the groups was significant $(P<0.05)$. However, the influx of ${ }^{131}$ I-albumin $\left(K_{i}=0.47 \pm 0.16 \mu \mathrm{l} /\right.$ gmin) in the alcohol group tended $(P=0.055)$ to be higher than in the control group $(0.03 \pm 0.13 \mu \mathrm{l} / \mathrm{gmin})$. After subtraction of the vascular space to normalize the actual entry of leptin, the influx rate was $0.37 \pm 0.17 \mu \mathrm{l} /$ gmin for the control group and $0.76 \pm 0.26 \mu \mathrm{l} / \mathrm{gmin}$ for the alcohol group. The difference between the two groups was not significant. Thus, alcohol feeding increased leptin influx, and this increase was associated with a partial disruption of the BBB as shown by a trend toward increased albumin influx (Figure 2a).

Despite an increase in the general permeability of the BBB to albumin associated with the influx of leptin, as shown above, the saturable transport system for leptin was still functional. This was evident after i.v. injection and multiple-time regression analysis in alcohol-fed mice receiving the ${ }^{125}$ I-leptin tracer in the presence or absence of excess unlabeled leptin. As shown in Figure 2b, excess leptin $(1 \mu \mathrm{g} /$ mouse) significantly decreased the uptake of ${ }^{125}$ I-leptin ( $n=9 /$ group), showing saturable transport.

The Effect of Forced Alcohol Diet on the Blood-to-Brain Influx of Albumin (Vascular Marker) and Leptin (Polypeptide with Specific Transport System) in Alcohol-Preferring B6 Mice

In situ brain perfusion was performed in groups of B6 mice 2 weeks after feeding of the alcohol or control liquid diet. Like the CD1 mice, the influx rates of both ${ }^{125}$ I-leptin and

${ }^{131}$ I-albumin in the B6 mice were higher in the alcohol-fed group. The value of [ ${ }^{125} \mathrm{I}$-leptin- ${ }^{131} \mathrm{I}$-albumin] was calculated to determine the amount of leptin completely crossing the $\mathrm{BBB}$ and taken up in the brain parenchyma by selective entry, without interference from the vascular space. The alcohol group had a corrected $K_{\mathrm{i}}$ of $0.31 \pm 0.07 \mu \mathrm{l} / \mathrm{gmin}$ $(n=11)$, whereas that in the control group was $0.15 \pm 0.04 \mu \mathrm{l} /$ gmin $(n=13)$. The difference between the groups was significant $(P<0.05)$ (Figure $3 a)$. As the $\mathrm{BBB}$ was partially disrupted, this differential permeability shows that the increase of leptin influx exceeded that of albumin influx, suggesting an upregulated transport system component to the increased leptin influx. Comparison of the $K_{\mathrm{i}}$ of leptin in the nonalcohol B6 and CD1 mice showed that the inbred B6 mice $(n=10)$ had a lower $K_{\mathrm{i}}$ than the outbred CD1 mice $(n=6)$ (Figure 3b). Regardless, the increase of leptin influx in the B6 mice after alcohol exceeded that of barrier disruption, probably reflecting upregulation of the transport system.

\section{Paired Feeding Study Showed that a Lower Level of Alcohol Drinking did not Cause BBB Disruption or Increased Influx of Leptin}

Two identical bottles of control diet were provided to the B6 mice, and spatial bias (preference for drinking from a particular location) was avoided by rotation of the bottles of food and water every other day. The 14-day study, the same duration as in the forced alcohol study, began when one of the two bottles in the randomly chosen alcohol group was switched to $5 \%$ alcohol at day 0 . As shown in Figure 4a, on day 0 , all mice were fed the control diet. The first preference for the alcohol or control diet was recorded on day 2 . Thereafter, the mice in the alcohol-fed group showed significantly greater $(P<0.005)$ intake of the control liquid diet than the alcohol liquid diet on each study day. At the 

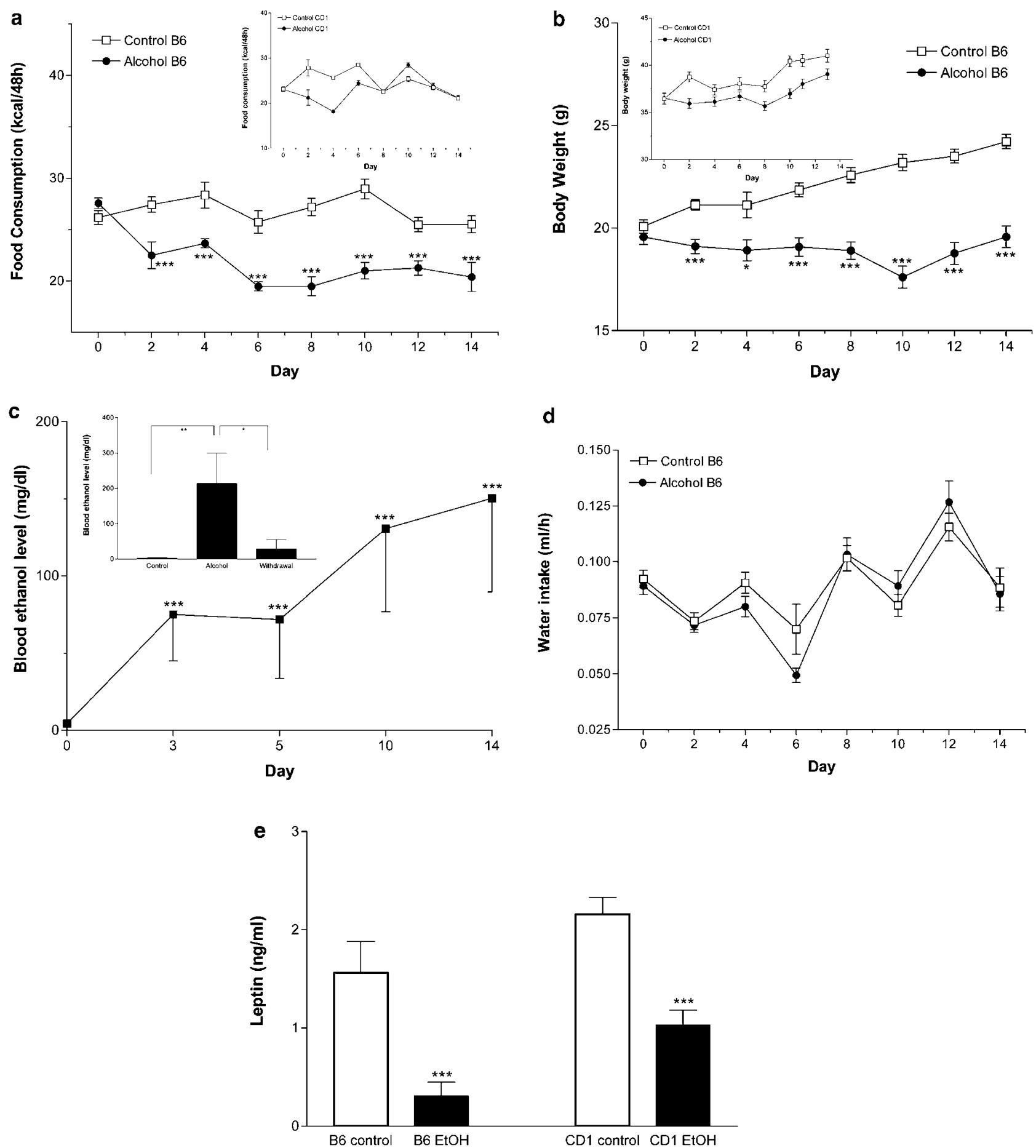

Figure I Effects of alcohol diet on food and water consumption, weight gain, blood-alcohol, and -leptin levels. (a) A significant decrease of food consumption was seen in alcohol-fed mice as compared with the control-fed mice. The study with B6 mice used I2-I4 mice/group, and that with CDI mice used $7 /$ group (inset). Measurements were taken every other day. ${ }^{*} * *<<0.005$. (b) The alcohol diet was associated with significantly lower body weights for both B6 and $\mathrm{CDI}$ (inset) mice as compared with those fed the isocaloric control diet. Measurements were taken every other day ( $n=7-\mid 4 / g r o u p)$. $* P<0.05$; **** $P<0.005$. (c) In CDI mice fed the alcohol diet, blood-alcohol concentrations showed a stepwise increase over the course of 2 weeks ( $n=5-$ I0/time point). In B6 mice studied with the same diet, the alcohol levels were even higher but decreased rapidly after 2 days of withdrawal (inset). $* P<0.05$ ** $P<0.01$; **** $P<0.005$ compared with the baseline. (d) Water consumption did not show differences in groups of mice fed with the control diet or alcohol diet. Measurements were taken every other day ( $n=14 /$ group). (e) Blood-leptin concentrations measured at day 14 in mice on a forced alcohol diet showed a significant decrease (*** $P<0.005)$ compared with the control groups in both B6 and CDI strains of mice ( $n=6-8 /$ group).

end of the study, the weight gain in the alcohol group (offered both control and alcohol diets) was similar to that in the control groups (offered two identical bottles of control diet) (Figure $4 \mathrm{~b}$ ). BBB transport assays at the end of the study showed that the uptake of leptin and albumin was not significantly different between the two groups (Figure 4c). Because of anticipated large individual variations in alcohol drinking, 7 mice were included for each of 

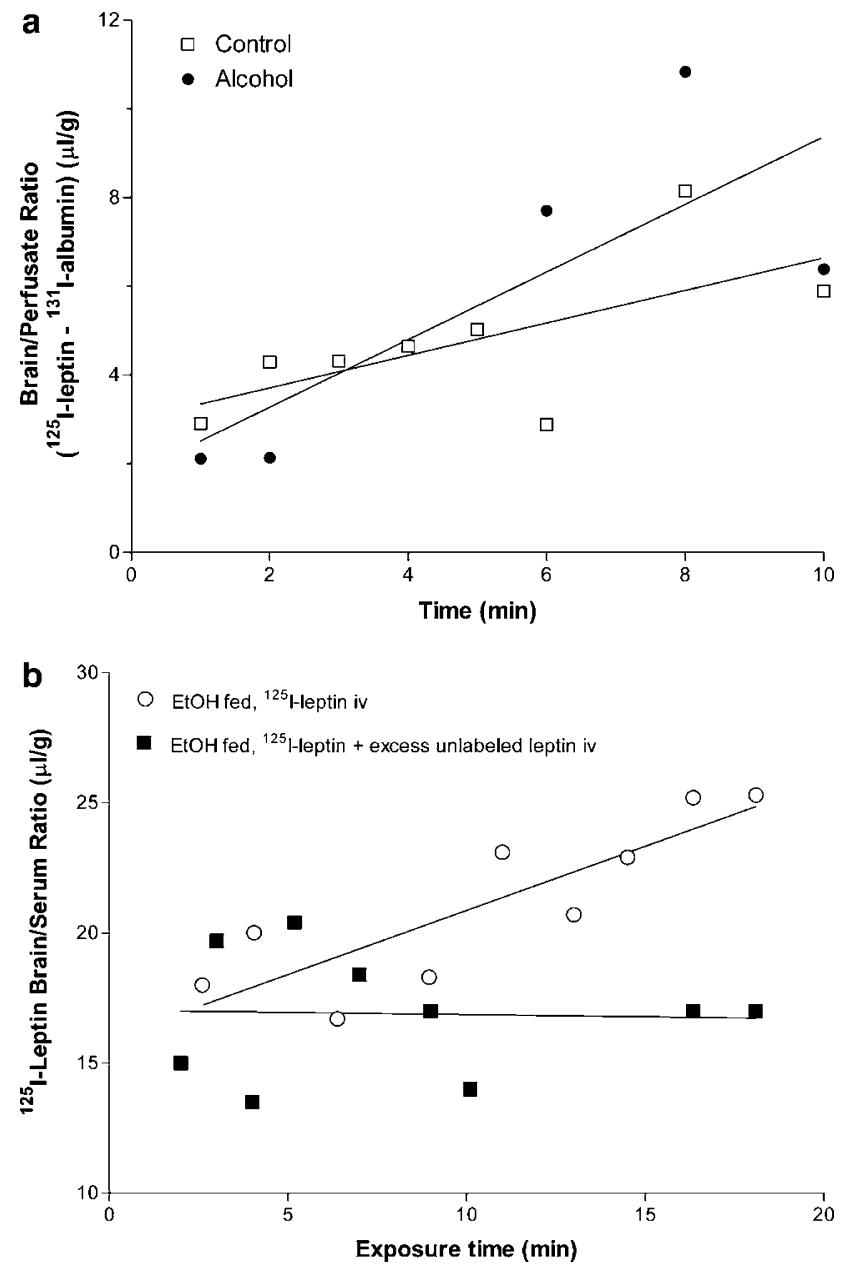

Figure 2 Effects of alcohol feeding on the BBB permeability in CDI mice. (a) In situ brain perfusion showed that nonalcohol-preferring CDI mice receiving a forced alcohol diet had a significantly $(P<0.05)$ higher influx rate of ${ }^{125}$-leptin and a trend $(P=0.055)$ toward increased ${ }^{131}$ I-albumin that was injected simultaneously ( $n=7-8 /$ group). (b) After i.v. injection of ${ }^{125}$-leptin, its influx rate from blood to brain was significantly $(P<0.05)$ decreased by addition of excess unlabeled leptin in $C D I$ mice on the forced alcohol diet for 14 days, showing that saturability of the BBB transport system remained ( $n=9 /$ group).

the control diet groups and 12 mice were used for each of the alcohol groups in this study.

\section{Acute Alcohol Administration did not Modulate BBB Permeability to Leptin Nor did it Disrupt the BBB}

Groups of mice were studied $4 \mathrm{~h}$ after i.p. injection of $20 \%$ alcohol $(2 \mathrm{~g} / \mathrm{kg})$ or normal saline control ( $n=6 /$ group). In each group, the brain/perfusate ratio of ${ }^{125}$ I-leptin and ${ }^{131}$ I-albumin after $5 \mathrm{~min}$ of in situ brain perfusion was calculated. In neither $\mathrm{CD} 1$ nor $\mathrm{B} 6$ mice, was there any significant change in the brain uptake of albumin (indicative of vascular space and potential $\mathrm{BBB}$ disruption) or leptin (polypeptide crossing the BBB with a specific transport system) (Figure 5). Studies of the $\mathrm{CD} 1$ and B6 mice were conducted on different days with different batches of radioisotopes.
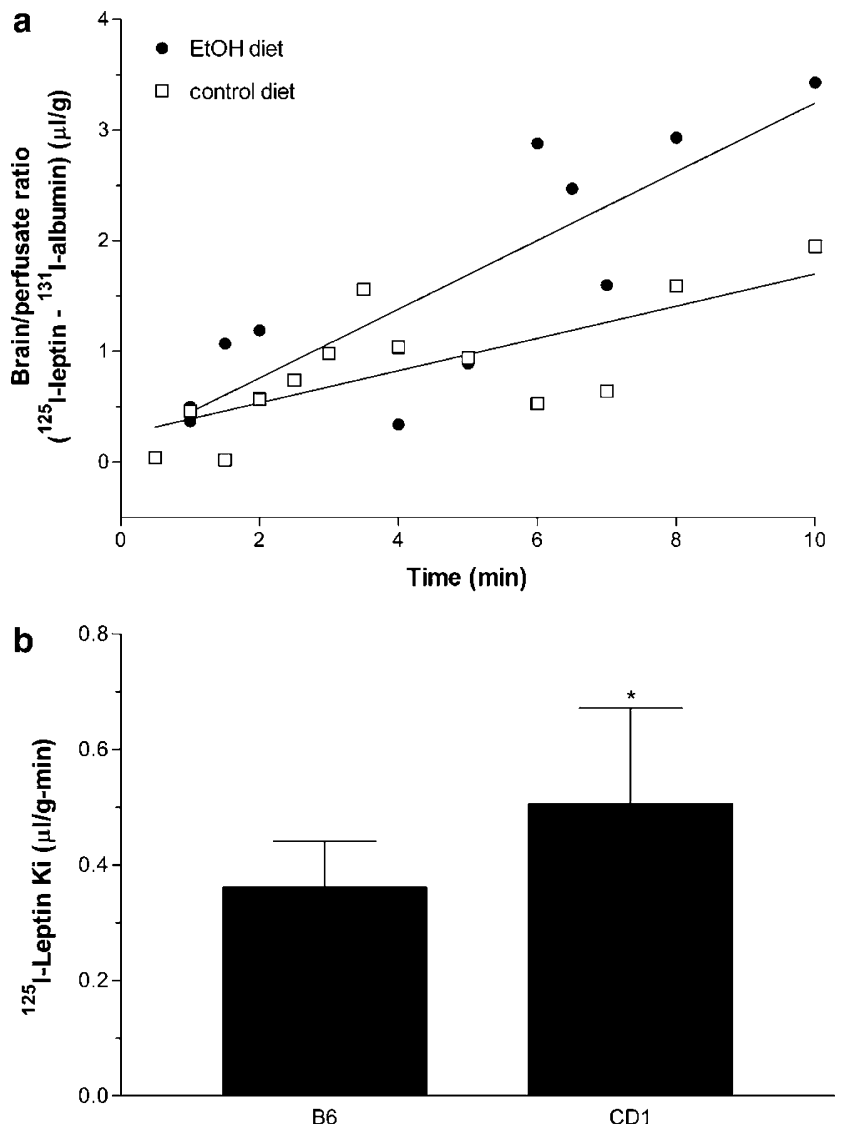

Figure 3 Effects of alcohol diet on BBB permeability of the B6 mice, which had a lower basal influx rate for leptin. (a) In situ brain perfusion showed that alcohol-preferring B6 mice fed a forced alcohol diet for 14 days had a significantly $(n=11, P<0.05)$ higher ${ }^{125}$-leptin influx (corrected by subtraction of albumin influx) than mice fed the isocaloric control diet $(n=13)$. (b) Comparison from different studies showed that CDI mice had a higher $K_{i}$ for leptin across the BBB than the B6 mice. Results were derived from six independent studies. $* P<0.025$.

\section{DISCUSSION}

Alcohol readily crosses the BBB because of its high lipid solubility (Raichle et al 1976). However, the effects of alcohol on the permeability of the BBB in adult mammals have been inconsistent. Earlier studies, mostly in the 1980's, showed three types of results: increased permeability with evidence of structural damage (Karwacka, 1980; Gulati et al, 1985; Stewart et al, 1988; Borisenko, 1990); no change induced by alcohol (Hemmingsen et al, 1980; Hillbom and Tervo, 1981; Phillips, 1981; Phillips and Cragg, 1982); and even a protective and beneficial effect (Neilly and Lin, 1986). The conflicting results are not easily reconciled by dose, duration, or other pharmacological factors, whereas a detrimental effect of alcohol on tight junction proteins and alcohol-induced oxidative stress on endothelium was evident in a cell-culture system (Haorah et al, 2005b).

Therefore, we further tested the relationship between leptin permeation and alcohol drinking by use of different strains of mice, different doses of alcohol, and different durations of administration. Forced alcohol feeding for 2 weeks was associated with reduced food consumption and weight loss, even in alcohol-preferring B6 mice. This is similar to clinical observations in chronic alcoholic patients. 

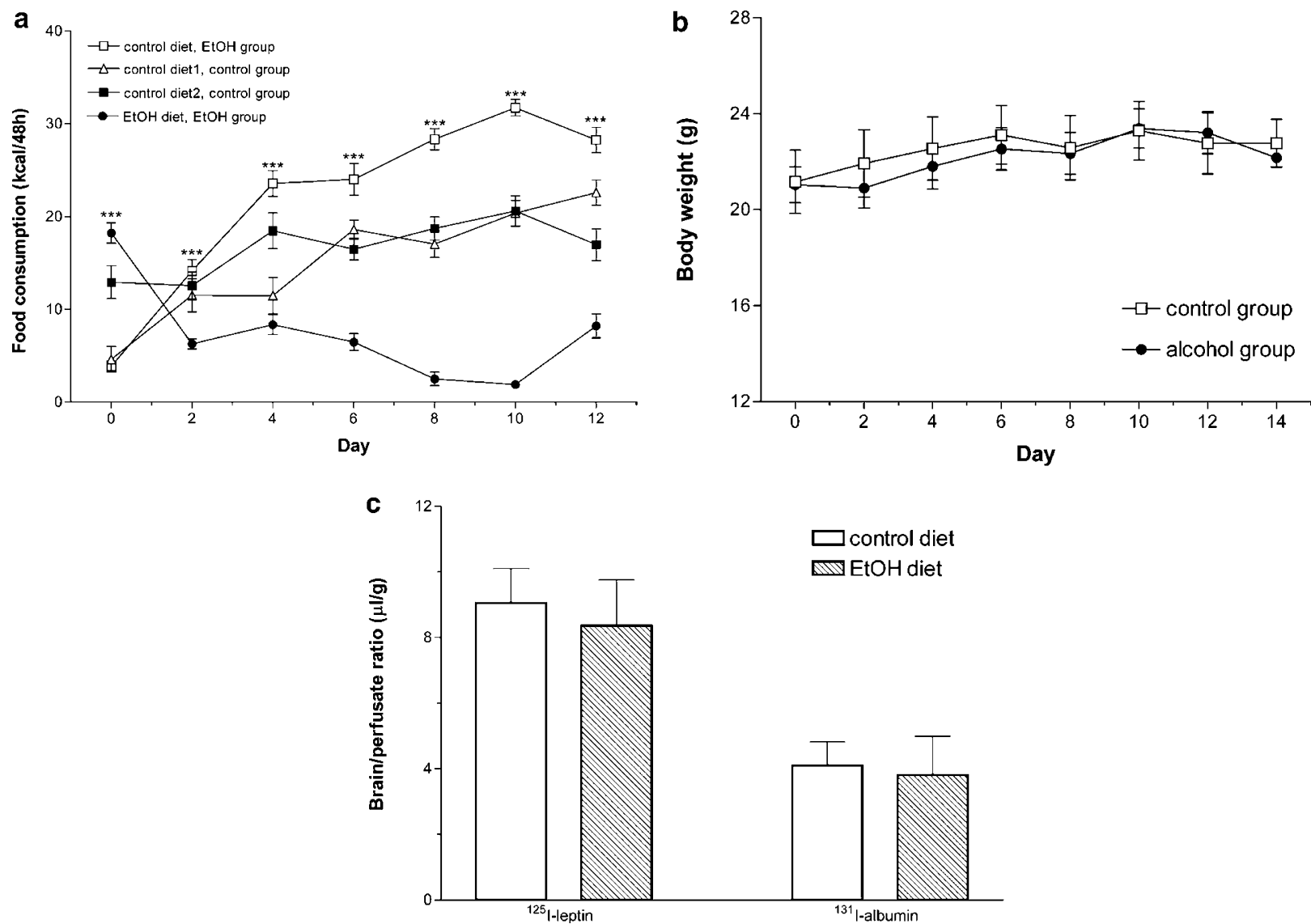

Figure 4 Effects of alcohol choice diet on food preference, body weight, and BBB permeability. (a) During the two-bottle choice study, the alcoholpreferring strain of B6 mice still preferred the control liquid diet $(n=7)$ more than the alcohol diet $(n=12)$. *** $P<0.005$. (b) The group of mice given the choice of consuming either alcohol or isocaloric liquid control diet had a similar weight gain as the group of control mice fed an isocaloric diet without alcohol. (c) Mice with the alcohol choice feeding showed no significant changes in leptin or albumin permeation across the BBB after I4 days.

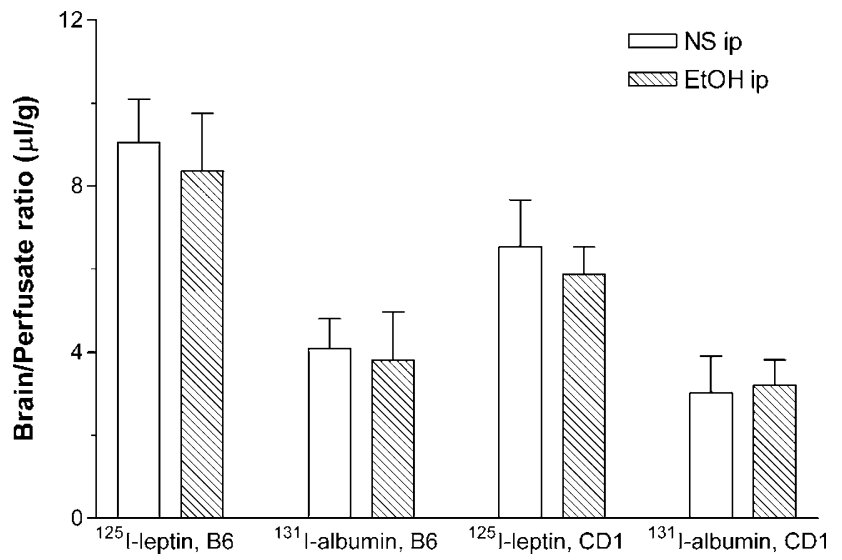

Figure 5 Acute treatment with alcohol $(20 \%$ at $2 \mathrm{~g} / \mathrm{kg})$ vs normal saline (NS) by i.p. injection did not cause a significant change in the brain uptake of ${ }^{125}$ I-leptin or ${ }^{131}$ I-albumin. The radiotracers were administered simultaneously for $5 \mathrm{~min}$ by in situ brain perfusion in either B6 or CD I mice ( $n=6 /$ group).

Although we cannot rule out taste aversive effects, the blood concentration of alcohol reached a level considered as intoxication, indicating that the chronic ingestion model was successful. Correlated with the lower body weight, the alcohol group had significantly decreased blood-leptin concentrations.
After in situ brain perfusion, the influx rate of ${ }^{125}$ I-leptin was significantly higher in the alcohol-fed mice than the controls. This increased influx could indicate that: (1) the transport system functions at a higher capacity; (2) there was disruption of the $\mathrm{BBB}$ in the alcohol-fed mice; or (3) both processes are involved. By inclusion of excess unlabeled leptin to show saturability, we demonstrated that the transport system for leptin persisted in the alcohol-fed mice. By including simultaneous administration of the vascular marker ${ }^{131} \mathrm{I}$-albumin in the studies, we also showed partial BBB disruption in mice when alcohol was the main supply of energy intake for 2 weeks. The inert protein albumin serves as a permeability marker as its basal penetration across the intact BBB is low. With these studies, we showed that the effects of alcohol were dose- and timedependent, as increased BBB permeation was not seen in mice provided with two-bottle choice feeding for the same duration, or in mice receiving a large dose of alcohol $4 \mathrm{~h}$ before the end of the study. Thus, coexisting BBB disruption and persistent leptin transport contributed to the overall influx of leptin in alcohol-ingesting mice.

In support of the observation that alcohol increased the permeability of the $\mathrm{BBB}$, electron microscopic studies of cerebral capillary endothelia after chronic alcohol administration showed increased micropinocytic vesicles, mitochondria, smooth endoplasmic reticulum, and the Golgi apparatus, whereas the astrocytic processes reinforcing the 
BBB showed edema and abnormal mitochondria (Karwacka, 1980). Partial disruption of the BBB and increased nonspecific paracellular permeability are also consistent with the observation in vitro that alcohol treatment activates myosin light chain kinase, alters the distribution and function of the tight junction proteins occludin and claudin-5, and reduces transendothelial electrical resistance (Haorah et al, 2005a).

Both CD1 wild-type and B6 inbred mice adjusted to the diet smoothly during the adaptation phase involving a gradual increase of alcohol to $5 \%$ over 9 days. The blood concentration of leptin was lower in the B6 mice, and further reduction after alcohol ingestion was more pronounced in the B6 mice. The reduced serum leptin in the alcohol-ingesting mice was consistent with their lower body weight. A correlation between body weight and blood-leptin concentration has been well documented (McGregor et al, 1996). As both strains of mice showed increased uptake of leptin after ingestion of alcohol, the relatively smaller amount of leptin available to the brain in B6 mice might partially explain their reported higher tolerance to alcohol. Regardless, such preference appeared to be rather moderate, as B6 mice preferred to consume more of the control diet than the alcohol diet in the choice feeding study. The decreased serum leptin concentrations are probably explained by a lower production of leptin in the periphery, as the mice had slower weight gain than the controls. This is consistent with our observation from the transport assays that leptin permeation probably results from a combination of partial disruption of the $\mathrm{BBB}$ and persistent saturable transport. Transport of leptin across the $\mathrm{BBB}$ is unlikely to be the reason for the decreased serum leptin.

To determine whether the effect of alcohol on BBB permeability was immediate and direct or mediated by chronic adaptation, we tested the permeation of the BBB to albumin and leptin $4 \mathrm{~h}$ after alcohol administration by the i.p. route, an effective method to test acute alcohol actions (Redetzki et al, 1983; Broadbent et al, 2005). The lack of significant increase in the uptake of leptin and albumin within this short time suggests that the adaptive changes of the $\mathrm{BBB}$ might require a higher dose or longer duration of exposure.

The greater increased brain influx of leptin than albumin indicates the differential permeability of a partially disrupted $\mathrm{BBB}$. The normalized influx rate of $\left[{ }^{125} \mathrm{I}\right.$-leptin- ${ }^{131} \mathrm{I}-$ albumin] was significantly higher in mice after chronic alcohol ingestion. It suggests that both barrier disruption and an upregulated transport system were involved in the influx of leptin from blood to brain. Similar regulatory changes have been seen with other polypeptide cytokines in mouse models of autoimmune encephalomyelitis (Pan et al, 1996), brain and spinal cord injury (Pan et al, 1997, 2003, 2006a), and stroke (Pan et al, 2006b). As the blood concentration of leptin was significantly reduced in mice with chronic alcohol intoxication, the greater permeability of the BBB to leptin may serve to prevent further reduction of brain leptin concentrations to a premorbid level. Banks et al (1996, 2006a) has elegantly demonstrated the relevance of rodent studies to primates in terms of leptin transport (Farr et al, 2006; Rolland et al, 2006). Although the higher leptin influx may not improve the wasting syndrome seen in chronic alcoholism, homeostasis of the CNS feeding signal would be better maintained by such regulatory changes at the BBB.

In summary, increased leptin permeation across the $\mathrm{BBB}$ occurred concomitantly with BBB disruption after 2 weeks of forced alcohol ingestion. This increase was not seen when the mice had a choice of a nonalcohol diet or when alcohol was given acutely as a single high dose. Thus, increased BBB permeability to leptin was a consequence of prolonged alcohol intake. The mechanisms involved include both BBB disruption and the persistence of a specific transport system.

\section{ACKNOWLEDGEMENTS}

We thank many members of the BBB Lab for assistance in generating preliminary data, and Ms Loula Burton for editorial support. Support for WP was provided by NIH grants NS45751 and NS46528. Support for AJK was provided by NIH grants AA12865 and DK54880.

\section{DISCLOSURE/CONFLICT OF INTEREST}

The authors declare that, except for income received from my primary employer, no financial support or compensation has been received from any individual or corporate entity over the past 3 years for research or professional service and there are no personal financial holdings that could be perceived as constituting a potential conflict of interest.

\section{REFERENCES}

Balasubramaniyan V, Nalini N (2006). Exogenous leptin improves insulin sensitivity in ethanol fed insulin resistant mice. Clinica Chimica Acta 367: 201-203.

Banks WA, Clever CM, Farrell CL (2000). Partial saturation and regional variation in the blood-to-brain transport of leptin in normal weight mice. Am J Physiol 278: E1158-E1165.

Banks WA, Farr SA, Morley JE (2006a). The effects of high fat diets on the blood-brain barrier transport of leptin: failure or adaptation? Physiol Behav 88: 244-248.

Banks WA, Jaeger LB, Urayama A, Kumar VB, Hileman SM, Gaskin FS et al (2006b). Preproenkephalin targeted antisenses cross the blood-brain barrier to reduce brain methionine enkephalin levels and increase voluntary ethanol drinking. Peptides 27: 784-796.

Banks WA, Kastin AJ, Huang W, Jaspan JB, Maness LM (1996). Leptin enters the brain by a saturable system independent of insulin. Peptides 17: 305-311.

Banks WA, Niehoff ML, Martin D, Farrell CL (2002). Leptin transport across the blood-brain barrier of the Koletsky rat is not mediated by a product of the leptin receptor gene. Brain Res 950: $130-136$.

Belknap JK, Crabbe JC, Young ER (1993). Voluntary consumption of alcohol in 15 inbred mouse strains. Psychopharm 112: 503-510.

Blednov YA, Walker D, Harris RA (2004). Blockade of the leptinsensitive pathway markedly reduces alcohol consumption in mice. Alcohol Clin Exp Res 28: 1683-1692.

Borisenko SA (1990). Effects of drugs on blood-brain barrier permeability in rats chronically intoxicated by ethanol. Ann Ist Super Sanita 26: 39-42. 
Broadbent J, Kampmueller KM, Koonse SA (2005). Role of dopamine in behavioral sensitization to ethanol in DBA/2J mice. Alcohol 35: 137-148.

Farr SA, Banks WA, Morley JE (2006). Effects of leptin on memory processing. Peptides 27: 1420-1425.

Gulati A, Nath C, Shanker K, Srimal RC, Dhawan KN, Bhargava KP (1985). Effect of alcohols on the permeability of blood-brain barrier. Pharmacol Res Commun 17: 85-93.

Haorah J, Heilman D, Knipe B, Chrastil J, Leibhart J, Ghorpade A et al (2005a). Ethanol-induced activation of myosin light chain kinase leads to dysfunction of tight junctions and blood-brain barrier compromise. Alcohol Clin Exp Res 29: 999-1009.

Haorah J, Knipe B, Leibhart J, Ghorpade A, Persidsky Y (2005b). Alcohol-induced oxidative stress in brain endothelial cells causes blood-brain barrier dysfunction. J Leukocyte Biol 78: 1223-1232.

Hemmingsen R, Hertz MM, Bolwig TG (1980). Integrity of bloodbrain barrier during ethanol intoxication and withdrawal in the rat: normal glucose transfer and permeability to $\mathrm{Na}^{+}$and $\mathrm{Cl}^{-}$. Stroke 11: 141-144.

Hillbom ME, Tervo TM (1981). Ethanol and acetaldehyde do not increase the blood-brain and blood-retinal barrier permeability to sodium fluorescein. Experientia 37: 936-938.

Karwacka H (1980). Ultrastructural and biochemical studies of the brain and other organs in rats after chronic ethanol administration. I. Electronmicroscopic investigations of the morphologic elements of the blood-brain barrier in the rat after ethanol intoxication. Exp Pathol 18: 118-126.

Kastin AJ, Akerstrom V, Pan W (2001). Validity of multiple-time regression analysis in measurement of tritiated and iodinated leptin crossing the blood-brain barrier: meaningful controls. Peptides 22: 2127-2136.

Kastin AJ, Pan W (2003). Feeding peptides interact in several ways with the blood-brain barrier. Curr Pharm Des 9: 789-794.

Kastin AJ, Pan W (2006). Editorial: intranasal leptin: blood-brain barrier bypass (BBBB) for obesity? Endocrinology 147: 2086-2087.

Kastin AJ, Pan W, Maness LM, Koletsky RJ, Ernsberger P (1999). Decreased transport of leptin across the blood-brain barrier in rats lacking the short form of the leptin receptor. Peptides 20: 1449-1453.

McGregor GP, Desaga JF, Ehlenz K, Fischer A, Heese F, Hegele A et al (1996). Radiommunological measurement of leptin in plasma of obese and diabetic human subjects. Endocrinology 137: 1501-1504.

Neilly JP, Lin JC (1986). Interaction of ethanol and microwaves on the blood-brain barrier of rats. Bioelectromagnetics 7: 405-414.
Pan W, Akerstrom V, Zhang J, Pejovic V, Kastin AJ (2004). Modulation of feeding-related peptide/protein signals by the blood-brain barrier. J Neurochem 90: 455-461.

Pan W, Banks WA, Kastin AJ (1997). BBB permeability to ebiratide and TNF in acute spinal cord injury. Exp Neurol 146: 367-373.

Pan W, Banks WA, Kastin AJ (1998). Permeability of the bloodbrain/spinal cord barrier to neurotrophins. Brain Res 788: 87-94.

Pan W, Banks WA, Kennedy MK, Gutierrez EG, Kastin AJ (1996). Differential permeability of the BBB in acute EAE: enhanced transport of TNF-a. Am J Physiol 271: E636-E642.

Pan W, Cain C, Yu Y, Kastin AJ (2006a). Receptor-mediated transport of LIF across blood-spinal cord barrier is upregulated after spinal cord injury. J Neuroimmunol 174: 119-125.

Pan W, Ding Y, Yu Y, Ohtaki H, Nakamachi T, Kastin AJ (2006b). Stroke upregulates TNF alpha transport across the blood-brain barrier. Exp Neurol 198: 222-233.

Pan W, Kastin AJ, McLay RN, Rigai T, Pick CG (2003). Increased hippocampal uptake of TNFa and behavioral changes in mice. Exp Brain Res 149: 195-199.

Phillips SC (1981). Does ethanol damage the blood-brain barrier? J Neurol Sci 50: 81-87.

Phillips SC, Cragg BG (1982). Weakening of the blood-brain barrier by alcohol-related stresses in the rat. J Neurol Sci 54: 271-278.

Raichle ME, Eichling JO, Straatmann MG, Welch MJ, Larson KB, Ter Pogossian MM (1976). Blood-brain barrier permeability of 11C-labeled alcohols and 15O-labeled water. Am J Physiol 230: 543-552.

Redetzki JE, Griswold KE, Nopajaroonsri C, Redetzki HM (1983). Amelioration of cardiotoxic effects of alcohol by vitamine $\mathrm{E}$. J Toxicol Clin Toxicol 20: 319-331.

Rolland YM, Perry HM, Patrick P, Banks WA, Morley JE (2006). Leptin and adiponectin levels in middle-aged postmenopausal women: associations with lifestyle habits, hormones, and inflammatory markers - a cross-sectional study. Metabolism 55: $1630-1636$.

Stewart PA, Hayakawa EM, Carlen PL (1988). Ethanol and pentobarbital in combination increase blood-brain barrier permeability to horseradish peroxidase. Brain Res 443: 12-20.

Urayama A, King K, Gaskin FS, Farr SA, Banks WA (2006). Effects of chronic ethanol administration on brain interstitial fluid levels of methionine-enkephalin as measured by microdialysis in vivo. Peptides 27: 2201-2206.

Vavrousek-Jakuba EM, Baker PA, Shoemaker WJ (1991). Effect of ethanol on maternal and offspring characteristics: comparison of three liquid diet formulation fed during gestation. Alcohol Clin Exp Res 15: 129-135. 